\title{
Correction of Transposition of Great Arteries with Ventricular Septal Defect and Left Outflow Tract Obstruction with Double Arterial Translocation with Preservation of the Pulmonary Valve
}

\author{
Gláucio Furlanetto and Beatriz H. S. Furlanetto \\ Furlanetto Institute \\ Brazil
}

\section{Introduction}

Transposition of the great arteries (TGA) was first recognized by Mathew Bailie in 1797 . The term transposition of the aorta and pulmonary artery (PA) was applied by Farre in 1814 . Van Praagh proposed that TGA was one variety of malposition of the great arteries secondary to aberrations in conotruncal development. The other tipes of malposition include double-outlet right ventricle, double-outlet left ventricle, and anatomically corrected malposition. According the Congenital Heart Surgery Nomenclature and Database Project (James 2000), TGA always has discordant ventriculoarterial alignment such that the aorta arises entirely or in large party from the right ventricle (RV), the PA arises entirely or in large part above the left ventricle (LV) and concordant atrioventricular alignment is nearly always present. The term simple TGA has come to be used to denote those patients without associated ventricular septal defect (VSD) or left ventricular outflow tract obstruction (LVOTO) and nearly $75 \%$ of TGA patients have the simple type. Usually in the simple form of TGA the left ventricle doesn't has subpulmonary conus, so there is fibrous continuity between pulmonary and mitral valves, and tha aorta is anterior and to the right of the PA. The VSD occur in $40-45 \%$ of TGA patients and about $30 \%$ of these defects will be very small. LVOTO occurs in $25 \%$ of patients and is rare in patients with intact ventricular septum, ocurring in 5\%. Approximated $30 \%$ of patients with TGA-VSD have LVOTO. A subvalvar LVOTO can be dynamic, localized fibrous ring, diffuse tunnel-like obstruction, muscular obstruction related to malposition of the outlet septum and LVOTO result from malposition of the mitral apparatus on the interventricular septum.

TGA is the second more common cyanotic congenital heart disease (CHD) and represents approximately 5-7\% of all CHD and has a incidence of 20-30 in 100.000 live births, with a male preponderance of approximately 2:1. In patients with TGA, VSD and LVOTO early survival reachs $70 \%$ at one year and $29 \%$ at 5 years. Neonates with TGA, VSD and severe LVOTO have diminished pulmonary blood flow and they represents 5-8\% of neonatal TGA population. Clinical findings are similar to those in the infants with tetralogy of Fallot with severe pulmonar stenosis. 
The physiologic abnormalitie in TGA is that systemic and pulmonary circulations function in parallel rather in series as in normal infance, the consequence is deficiency of oxygen supply to the tissues. The arterial oxigen saturation and the extend of intercirculatory mixing dependents on the number, size and position of the anatomic communications like atrial septal defect (ASD), VSD and persistent ductus arteriosus.

Infants with TGA, VSD and LVOTO have been successfully palliated with systemic-topulmonary anastomosis with a politetrafluorethilene (PTFE) modified Blalock-Taussig shunt. In these patients the intracardiac correction is carried out at later age. The optimal age for the performance of a corretive repair remains controversial because of the balance between palliation and correction. Palliation leads to the performance of Rastelli repair at an older age with larger conduits, therefore reducing the need for reoperations. However, palliation also leads to LV overloading, and cyanosis.

The conventional surgical technique to repair TGA, VSD and LVOTO is the Rastelli operation (Rastelli, 1969). This operation was described with the theoretic advantage of incorporating the left ventricle as the systemic ventricle for correction of TGA. This technique achieves redirection of the left ventricular outflows and relieves pulmonary stenosis by bypassing it. The repair consists of: 1) repair of the VSD with a patch in such a way to connect the LV with the aorta using the closure of the right ventricle outflow as part of the left ventricular outflow, 2) division of the pulmonary artery and oversewn the cardiac end and 3) reconstruction of the pulmonary artery with a valved conduit. However, the Rastelli operation is far from ideal because it is not feasible in many patients because of unfavorable intracardiac anatomy, it requires the use of a prosthetic conduit for the reconstruction of the pulmonary outflow tract and the intraventricular tunnel used at Rastelli operation is not ideal because it can show some degree of stenosis at medium follow-up.

The Lecompte procedure, or "reparation a l'etage ventriculaire" (Borromée, 1988) is a surgical procedure that resects the infundibular septum to creates a large communication between the LV and the aorta. The aim of this resection is to construct a straight and direct aortic outflow tract to the aortic valve. The aorta is connected to the LV with a patch smaller than in the Rastelli operation and the tunnel between the LV and aorta is situated just beneath the aorta orifice and occupies very little space in the RV cavity. The pulmonary artery is transected above the pulmonary valve and is translocated onto the subaortic ventricular incision and the pulmonary outflow tract was completed with an anterior patch and a monocusp pericardial valve.

Double-outlet LV is conventionally correct with an intraventricular tunnel or with an extracardiac conduit when pulmonary stenosis is present. Pulmonary root translocation with the pulmonary valve to the RV is an important alternative surgical technique to correct this CHD (Chiavarelli, 1992).

This concept was used to correct TGA, VSD and LVOTO (Silva, 2000). In this procedure the pulmonary artery and its branches were dissected from the aorta and its posterior connections. The pulmonary root was dissected from the LV with the pulmonary valve. Than the VSD is closed, diverting the blood from the LV to the aorta like in the Rastelli operation and the pulmonary root is translocated to the RV. The follow-up of two children showed that the pulmonary root diameter can grown.

The Nikaidoh procedure (Nikaidoh, 1984) changed the concept to treat the LVOTO. In this procedure, after dissection of the aorta and the main pulmonary artery, the ascending aorta was totally mobilized with the aortic valve and the coronary arteries, the pulmonary artery is transected just above the pulmonary valve. The pulmonary annulus, subpulmonic fibrous 
tissue, and the crista supraventricularis is transected anteriorly into the superior corner of the VSD. The ascending aorta is moved posteriorly and sutured to the posterior pulmonary annulus. The large anterior defect is now closed, from the margin of the VSD to the aortic root. A large pericardial patch is utilized to reconstruct the continuity of the RV outflow without implant of pulmonary valve.

The Nikaidoh procedure was modified by $\mathrm{Hu}(\mathrm{Hu}, 2007)$. In this procedure the ascending aorta and the pulmonary trunk were transected and the coronary arteries were detached. The pulmonary root and the ascending aortic with the aorta valve were dissected out of ventricles. The subvalvar stenosis was relieved by resecting the conal septum, the VSD is closed and the detached ascending aorta with the aortic valve is translocated posteriorly and coronary arteries was reimplanted. After the Lecompte maneuver the pulmonary root is translocated anteriorly to the RV outflow. The pulmonary root is incised and a monocusp bovine jugular vein was used to enlarge the RV outflow tract.

Direct surgical relief of severe LVOTO depends on the anatomic type and severity of obstruction. In patients with mild LVOTO it can be resected and the Jatene arterial switch operation (Jatene, 1976) can be done.

Another surgical approach is the aortic root translocation plus arterial switch (BautistaHernandez, 2007). In this surgical technique proposed by del Nido a segment of the ascending aorta was transected from the right ventricle. The coronary arteries were excised as circular shape from the ascending aorta than the main pulmonary artery was transected. The ampliation of the LVOTO was made like the Nilaidoh procedure from the pulmonary annulus toward the VSD. The aortic autograft was sewn to the LV outflow and to the ascending aorta after the Lecompte maneuver. Reimplantation of the coronary arteries was performed into the neoaorta. The RV to pulmonary arteries continuity was established by a homograft.

We proposed a new approach for correction of TGA, VSD and LVOTO performing the double arterial translocation with preservation of the pulmonary valve. In this surgical technique the ascending aorta is translocated with the aortic valve and the coronary arteries to the left ventricle, after correction of left outflow tract obstruction and correction of the VSD, associated to pulmonary root translocation to the right ventricle, conserving integrally the pulmonary valve (Furlanetto, 2010).

\section{Double arterial translocation with preservation of the pulmonary valve, surgical technique}

This procedure was achieved employing cardiopulmonary bypass with hypothermia at $25^{\circ} \mathrm{C}$ and myocardial protection with warm induction blood cardioplegic solution at a proportion of 3:1 followed by hypothermic cardioplegic solution and modified ultrafiltration. Initially an incision inferior to the aortic valve was made, excising the ascending aorta (including aortic valve and coronary arteries) beneath the annular level of aortic valve from the right ventricle. Then we performed the excision of the pulmonary root, including pulmonary valve, beneath the annular level of the pulmonary valve from the left ventricle [figure 1]. After section of the infundibular septum in direction to the VSD, the LVOT and the VSD was closed with glutharaldeide-fixed bovine pericardium patch [figure 2]. The resulting gap of the aortic translocation was partially closed with fresh autologous pericardium. Finally the ascending aorta with the aortic valve and the coronary arteries were sutured into the left ventricle outflow and the pulmonary root with the pulmonary valve was sutured into the right ventricular outflow [figure 3]. 


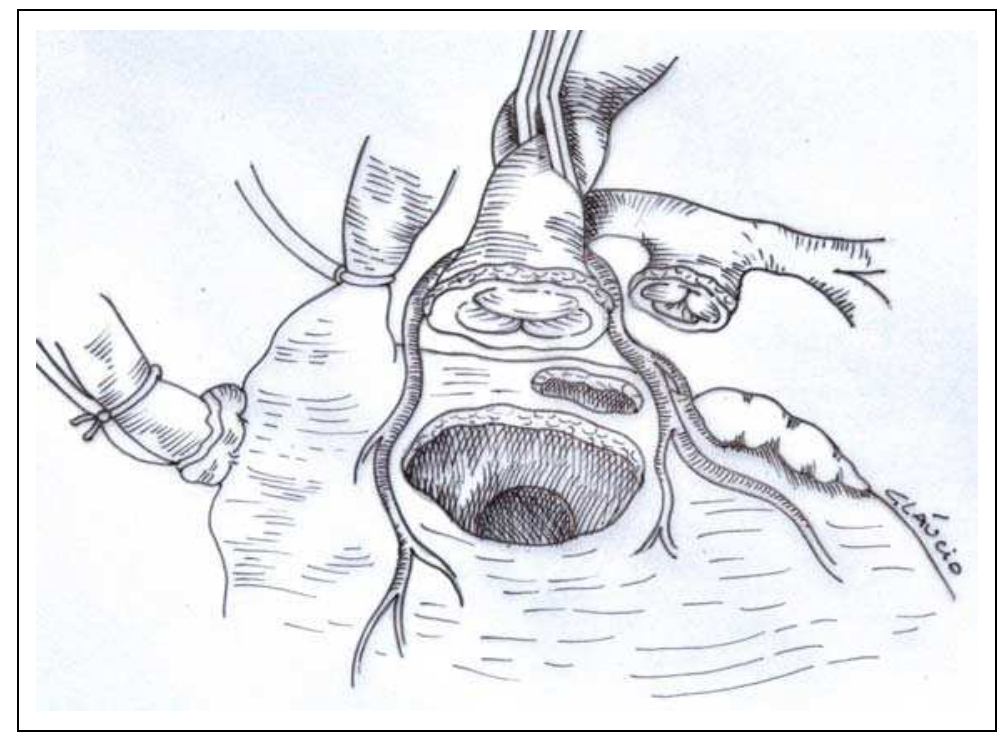

Fig. 1. Double arterial translocation with preservation of the pulmonary valve. Withdrawal of the ascending aorta with the aortic valve and coronary arteries from the right ventricle and removal of the pulmonary trunk with the pulmonary valve from the left ventricle

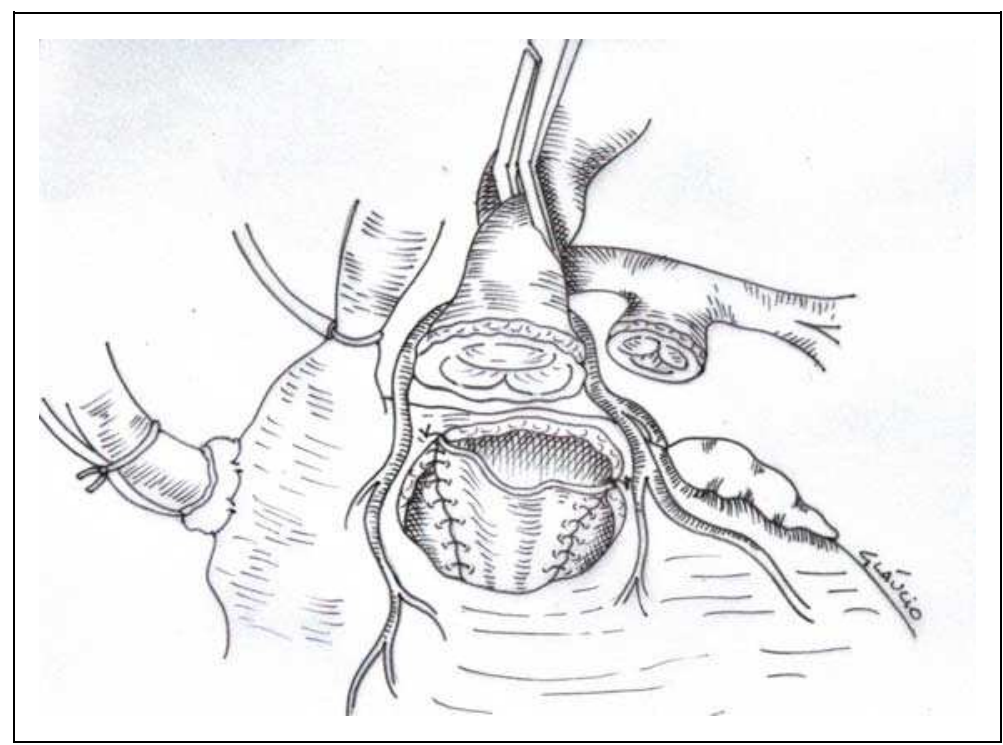

Fig. 2. Double arterial translocation with preservation of the pulmonary valve. After section of the pulmonary ring and infundibular septum toward the interventricular communication, an enlargement of the left ventricle outflow tract and a closure of interventricular communication was carried out using a glutaraldehyde-fixed bovine pericardium graft 


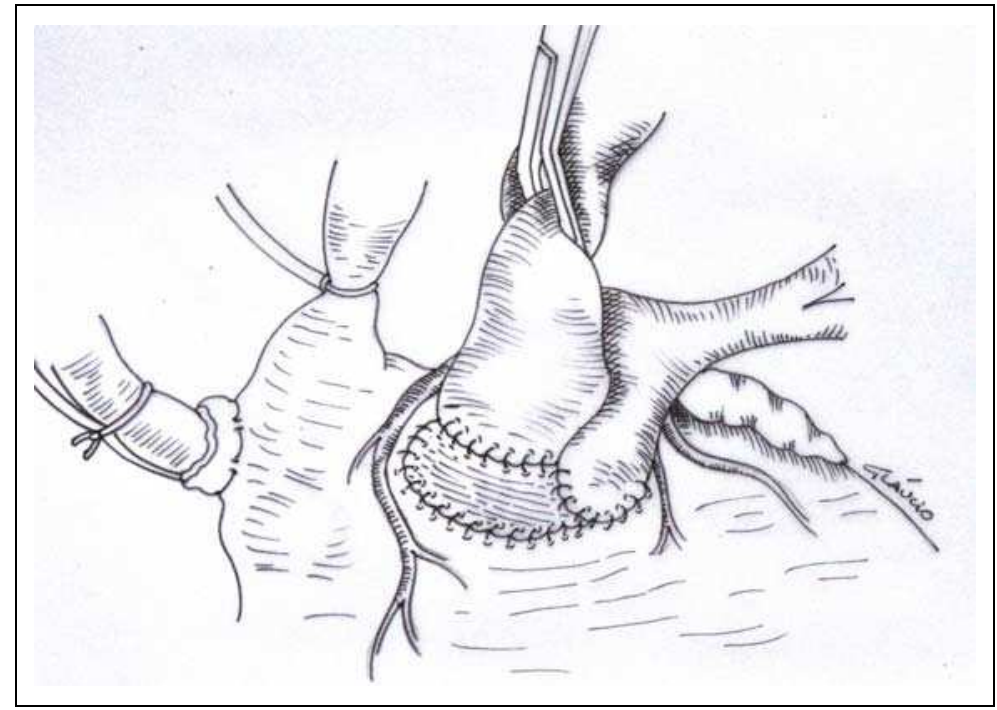

Fig. 3. Double arterial translocation with preservation of the pulmonary valve. The resulting opening from resection of the ascending aorta was partially closed to the right with a fresh autologous pericardial patch. The ascending aorta with the coronary arteries and the aortic valve was sutured to the left ventricle outflow tract and the pulmonary trunk with the pulmonary valve was sutured to the right ventricle outflow tract

\subsection{Patients, general}

Between November 1994 and June 2011, a total of 212 consecutive children with TGA were submitted to surgical treatment at the Hospital São Joaquim da Real e Benemérita Associação Portuguesa de Beneficência as follows: simple TGA ( $\mathrm{n}=110 ; 51,8 \%)$; TGA, VSD $(\mathrm{n}=66 ; 31,1 \%)$; TGA, VSD and LVOTO $(\mathrm{n}=34 ; 16,0 \%)$. The diagnosis was based on color echodopplercardiographic findings. The following procedures were used in children with TGA, VSD and LVOTO: modified BT shunt $(n=10)$, Rastelli procedure $(n=13)$, Jatene procedure $(n=3)$ and arterial translocation procedure $(n=8)$, with two further divisions modified Nikaidoh $(n=5)$ and double arterial translocation with preservation of pulmonary valve $(n=3)$.

In the modified BT shunt group, the VSD was not committed in two patients. Two patients had mitral valve straddling. Two patients had sistemic-pulmonar collateral arteries and one patient had multiple VSD. Seven patients were neonates, one was 2 months old and two patients were 1 year old. The mean weight was $4,3 \mathrm{~kg}$ (range $1,8 \mathrm{~kg}-8,8 \mathrm{~kg}$ ). In the Rastelli group three patients had previous modified BT shunt. Two patients had restrictive VSD. One patient had mitral valve insuficience and one patient had right pulmonary artery stenosis. Five patients were neonates, five patients were younger than 1 year of age (mean age was 6,2 months; range 2 months-eleven months), three patients were older than 1 year of age (mean age was 5,6 years; range 3 years - ten years). The mean weight was 7,9 kg (range $3,0 \mathrm{~kg}-28,0 \mathrm{~kg}$ ). In the Jatene procedure two patients were neonates and one patient was 4 months old. The mean weight was $3,8 \mathrm{~kg}$ (range $2,9 \mathrm{~kg}-5,2 \mathrm{~kg}$ ). In the arterial translocation group two patients had previous modified BT shunt and one patient had two 
previous modified BT shunt. Three patients were neonates, one patient was 6 months old and four patients were older than 1 year of age (mean age was 1,6 years; range 1 year - 2 years). The mean weight was 7,0 kg (range 2,6 kg - 11,3 kg).

\subsubsection{Patients, double arterial translocation with preservation of the pulmonary valve}

Three children with TGA with perimembranous VSD and LVOTO type-fibromuscular tubular obstruction associated to hypoplasia of the pulmonary valve ring underwent doble arterial translocation with preservation of the pulmonary valve: Patient 1 , female, aged two years, weighing $10.8 \mathrm{~kg}$, underwent two previous Blalock-Taussig shunt surgeries. Preoperative color Doppler echocardiography revealed a LVOTO gradient of $40 \mathrm{mmHg}$. CPB time was 195 minutes and the aortic clamping time was 123 minutes. Pressure measurement after surgical correction revealed a ratio of systolic pressure between the RV and LV (RV/LV) of 0.6 and a pulmonary gradient of $25 \mathrm{mmHg}$. The postoperative Doppler echocardiography revealed a pulmonary transvalvular gradient of $42 \mathrm{mmHg}$; Patient 2, male, aged 6 months, weighting $7.3 \mathrm{~kg}$. Preoperative Doppler echocardiography revealed LVOT gradient of 65 $\mathrm{mmHg}$. CPB time was 184 minutes and aortic clamping time was 140 minutes. Pressure measurement after surgical correction revealed RV/LV systolic pressure of 0.8 and pulmonary transvalvular gradient of $30 \mathrm{mmHg}$. Postoperative Doppler echocardiography revealed a pulmonary transvalvular gradient of $31 \mathrm{mmHg}$. Heart computaticional angiotomography (CT) performed during the immediate postoperative period showed appropriate positioning of the pulmonary trunk, pulmonary artery and aorta in both patients (Figure 4).

Patient 3, male, aged 1 year, weighting $8.0 \mathrm{~kg}$. Preoperative Doppler echocardiography revealed LVOT gradient of $31 \mathrm{mmHg}$. CPB time was 182 minutes and aortic clamping time was 155 minutes. Pressure measurement after surgical correction revealed RV/LV systolic pressure of 0.7 and pulmonary transvalvular gradient of $30 \mathrm{mmHg}$.

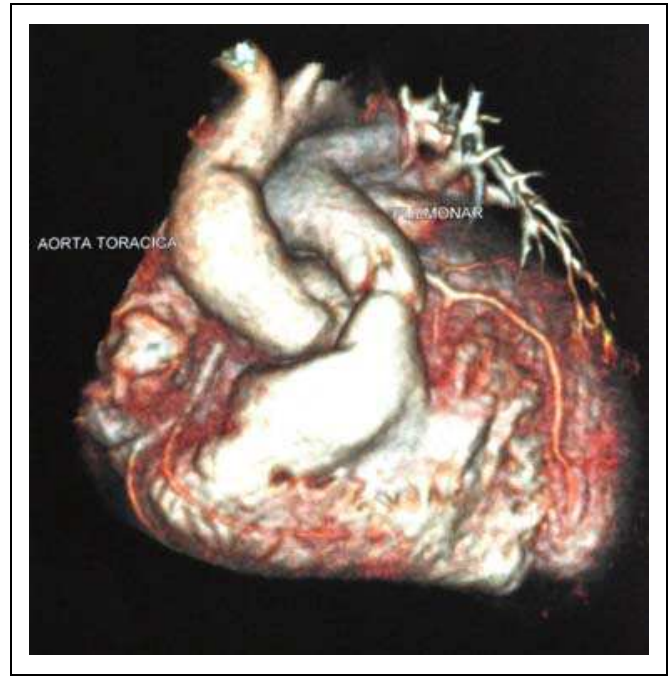

Fig. 4. Double arterial translocation with preservation of the pulmonary valve.

CT angiography performed in the immediate postoperative period showed appropriate positioning of the pulmonary artery and pulmonary branches as well as the ascending aorta 


\subsubsection{Surgical technique}

The palliative BT shunt was made by median sternotomy in seven patients, by right thoracotomy in two patients and by left thoracotomy in one patient. The interposition of a polytetrafluoroethylene (PTFE) tube was made between the innominate artery and the right pulmonary artery in nine patients, and between the left subclavian artery and the left pulmonary artery in one patient. In seven patients the diameter of the PTFE tube was $4 \mathrm{~mm}$, and in three patients it was $5 \mathrm{~mm}$. The Rastelli, Jatene and modified Nikaido procedures and double arterial translocation with preservation of the pulmonary valve were made by median sternotomy and moderate hypothermic cardiopulmonary bypass and myocardial protection with warm cardioplegic induction, followed by hypothermic blood cardioplegic solution at a proportion of 3:1 every 20 minutes. In the Rastelli procedure, the VSD was repaired through the right ventriculotomy in such a way to connect the left ventricle to aorta. The reconstruction of the pulmonary artery was performed with a glutaraldehydefixed bovine pericardium valved conduit in eight patients and with a glutaraldehyde-fixed bovine-valved jugular vein in six patients. In the Jatene group, the LVOTO was a subvalvar fibromuscular ridge. After aorta transection was performed resection of the localized LVOTO. The pulmonary trunk was transected proximal to its bifurcation. After the LeCompte maneuver, a button of the left and right coronary artery was excised from its sinus, and was inserted into the neoaorta. Coronary type was Yacoulb I in all patients. The reconstruction of the neopulmonar was done with a single, fresh autologous pericardium patch. In the modified Nikaidoh group the aorta was translocated with the aortic valve and coronary arteries to the left ventricle, after an enlargement of the left ventricle outflow tract and closure of VSD with a single glutaraldehyde-fixed preserved bovine pericardium patch. In this series of patients we used a valved conduit to reconstruct the right ventricular outflow. This approach was different from the original technique, in which the pulmonary trunk is used without a valve. We used a glutharaldeide-fixed valved bovine jugular vein in three patients. In one patient a glutharaldeide-fixed valved bovine pericardium conduit was used, and in one patient a Lhydro porcine valved pulmonary trunk [8]. During the analyzed period there was a change in the surgical procedure. At first, modified BT technique prevailed; later, Rastelli operation and Jatene operation were performed, and finally the arterial translocation operation.

\section{Results}

Two patients died in the modified BT shunt group (20\%), three patients died in the Rastelli group $(23,0 \%)$ and one patient died in the arterial translocation group $(20 \%)$. All patients in the Jatene group and in the double arterial translocation with preservation of the pulmonary valve all patients survived. The statistical analyses (Fisher's Exact Test) showed no difference between all groups $(p=0.811)$.

\section{Comments}

The surgical management of TGA with VSD and LVOTO is a surgical challenge. The Rastelli operation remains the most applied procedure for this congenital cardiopathy.

The most appropriate timing of the Rastelli operation is controversial. When it is performed during early infancy, it is a physiologic correction and avoids systemic hypoxemia. On the other hand, the palliative procedure of modified BT shunt performed during early infancy avoids early reoperation to change the valved conduit. 
There is a significant incidence of late mortality after Rastelli procedure, with survival rate of $82 \%$ at 5 years and $52 \%$ at 20 years. In 25 years of experience, Kreutzer noticed that the most common cause of late death was left ventricular failure, present in $25 \%$ of patients (Kreutzer, 2000). The intraventricular tunnel from left ventricle to aorta is tortuous and can develop stenosis in the late follow-up, which can explain the left ventricle disfunction. The presence of valved conduit dysfunction and right ventricular dysfunction may be associated with ventricular tachycardia and sudden cardiac death. Therefore, all patients submitted to Rastelli procedure will need to change the right ventricle to pulmonary artery conduit once. In the Lecompte procedure, the resection of the infundibular septum creates a straight, direct tunnel between the left ventricle to aorta occupying little space in the right ventricle outflow tract, in order to improve the intraventricular tunnel from the left ventricle to aorta. This procedure presents a better solution to the left ventricle outflow tract reconstruction, but in the right ventricle outflow the monocuspid pericardial valve used will develop some dysfunction on medium-term follow-up. The percentage of reoperation in patients submitted to LeCompte procedure is lower when compared to Rastelli procedure. A very interesting option to correct TGA, VSD and LVOTO is the Nikaidoh procedure. In this procedure, aortic translocation with the aortic valve and coronary arteries to the left ventricle, after enlargement of the left ventricular outflow and closure of the VSD with a single patch, creates an anatomical left ventricle outflow, but reconstruction of the right ventricle outflow without the pulmonary valve will cause right ventricle dysfunction. The $\mathrm{Hu}$ procedure utilizes the aortic translocation like the Nikaidoh procedure but translocating the pulmonary root and amplificating the pulmonary valve with a bovine jugular vein monocuspid to the right ventricle after performing the LeCompte maneuver performed to reconstruction of the right ventricle outflow. This procedure corrects the left ventricle outflow in an anatomical way, but the amplification of the pulmonary valve with a bovine jugular vein monocusp will certainly cause dysfunction of the pulmonary valve at mediumterm follow-up. The use of Silva procedure could avoid the reoperation to change the valved conduit. In this operation the pulmonary root is translocated with the pulmonary valve to the ventriculotomy on the right ventricle with expansion of the pulmonary root after dissection from the right ventricle. There is also the possibility of growth, because all the structures of the pulmonary root were preserved, but the correction of the left ventricle outflow tract is done like the Rastelli procedure with all disadvantages of this procedure. The surgery proposed by del Nido translocate a segment of the ascending aorta with anastomoses of the coronary arteries after enlargement of the left ventricle outflow tract employing a homograft to reconstruct the right ventricle outflow tract. Like in the Nikaidoh and $\mathrm{Hu}$ procedures the left outflow tract is anatomical but the use of a homograft at the right side will need change of the graft at medium follow-up.

The surgical technique that we performed, the double arterial translocation with preservation of the pulmonary valve, differs from all the other techniques used up to now because it corrects LVOTO through the aorta translocation with the aortic valve and coronary arteries after enlargement of the left outflow tract and closure of the VSD with a patch and corrects the right ventricle outflow with pulmonary root translocation with entire pulmonary valve to the right ventricle without the LeCompte maneuver and without right ventricolotomy. We believed that this procedure can be performed in patients with TGA, VSD and LVOTO when the pulmonary valve has an adequate annular diameter and the leaflets thickness is not too exacerbated. In these cardiopathies the LVOTO is subvalvar and valvar. The subvalvar stenosis is in the form of a localized fibrous ring, a tunnel-type 
fibromuscular or a muscular obstruction. The valvar stenosis is caused by annular hypoplasia and the valve could be bicuspid.

The use of prosthetic valves and of a connection without valve in the pulmonary position in children brings about dysfunction of the right ventricle on medium/late-term follow up. Based on this fact, the preservation of the native pulmonary valve with mild to moderate residual gradient has been accepted in the correction of tetralogy of Fallot by Voges (Voges 2008), who admit the size of the pulmonary valve with z-score superior to -4 . This concept can also be used in the conservation of the pulmonary valve performed at double arterial translocation with preservation of the pulmonary valve. It is possible that the pulmonary root could grow and the use of this surgical technique could diminish reoperation rates in these patients, but a late follow-up will be necessary to verify the potential of valve growth.

In one child submitted to double arterial translocation with preservation of the pulmonary valve the preoperative color doppler echocardiography revealed a LVOTO gradient of $40 \mathrm{mmHg}$. The transvalvar gradient between the right ventricle and the pulmonary trunk measured in the operating room after surgical correction was $25 \mathrm{mmHg}$, and the postoperative doppler echocardiography revealed a pulmonary transvalvar gradient of $42 \mathrm{mmHg}$. In another child, submitted to the same procedure, the preoperative color doppler echocardiography revealed a LVOTO gradient of $65 \mathrm{mmHg}$. The transvalvar gradient between the right ventricle and the pulmonary trunk measured in the operating room after surgical correction was $30 \mathrm{~mm} \mathrm{Hg}$ and the postoperative doppler echocardiography revealed a pulmonary transvalvar gradient of $31 \mathrm{mmHg}$. Heart computadorized angio-tomography performed during the immediate postoperative period showed appropriate positioning of the pulmonary trunk, the pulmonary artery and the aorta in both patients [Figure 4]. One third patient with preoperative Doppler echocardiography revealed a LVOT gradient of 31 $\mathrm{mmHg}$. The pressure measurement after surgical correction revealed RV/LV systolic pressure of 0.7 and pulmonary transvalvular gradient of $30 \mathrm{mmHg}$.

\section{Conclusion}

We can conclude that there was an evolution in the approach of TGA, VSD and LVOTO. The modified BT shunt operation, the operation of Rastelli and more recently the Jatene operation, modified Nikaidoh and the double translocation with preservation of the pulmonary valve were responsible for part of this evolution. In spite of the increase of surgical difficulty, there was no significant difference in the mortality. A late follow-up of a larger series of children will be important to check the potential development of the pulmonary valve in the double arterial translocation with preservation of the pulmonary valve.

\section{References}

Jaggers, J. J.; Cameron, D. E.; Herlong, R. Et al. (2000). Congenital Heart Surgery Nomenclature and Database Project: transposition of the great arteries. Ann Thorac Surg, Vol 69 (3) suppl 1, PP. 205-235.

Rastelli, C. ; Wallace, R.B. \& Ongley, A. (1969). Complete repair of transposition of the great arteries with pulmonary stenosis. A review and report of a case corrected by using a new surgical technique. Circulation, Vol.39, (Jannuary 1969), pp. 83-95. 
Borroomee, L.; Batisse, A.; Lecompte, Y. et al. (1988). Anatomic repair of anomalies of ventriculoarterial connection associated with ventricular septal defect. II. Clinical results in 50 patients with pulmonary outflow tract obstruction. J Thorac Cardiovasc Surg, Vol.95, (January 1988), pp. 96-102.

Chiavarelli, M.; Boucek, M.; Bailey, L. (1992). Arterial correction of double outlet left ventricle by pulmonary artery translocation. Ann Thorac Surg, Vol.53, (January 1992), pp. $1098-100$.

Silva, P.; Baumgratz, F.; Fonseca, L. (2000). Pulmonary root translocation in transposition of great arteries repair. Ann Thorac Surg, Vol.69, (February 2000), pp643-5.

Nikaidoh, H. (1984). Aortic translocation and biventricular outflow tract reconstruction. A new surgical repair for transposition of the great arteries associated with ventricular septal defect and pulmonary stenosis. J Thorac Cardiovasc Surg, Vol.88, (September 1984), pp. 365-72.

$\mathrm{Hu}$, S.; Li, J.; Wang, X. et al. (2007). Pulmonary and aortic root translocation in the management of transposition of the great arteries with ventricular septal defect and left ventricular outflow tract obstruction. J Thorac Cardiovasc Surg, Vol.133, (April 2007), pp. 1090-2.

Jatene, A.; Fontes, V.; Paulista, P. et al (1976). Anatomic correction of transposition of the great vessels. J Thorac Cardiovasc Surg, Vol.72, (March 1976), pp. 364-370.

Bautista-Hernandez, V.; Marx, G. R.; Bacha, E. A. et al. (2007). Aortic Root Translocation Plus Arterial Switch for Transposition of the Great Arteries With Left Ventricular Outflow Obstruction. JACC, Vol. 49, (January 2007), pp. 485-90.

Furlanetto, G.; Henriques, S.; Furlanetto, B. (2010). New technique: aortic and pulmonary translocation with preservation of pulmonary valve. Rev Bras Cir Cardiovasc, Vol.25, (January/March 2010), pp. 99-102.

Voges, I.; Fischer, G.; Scheewe, J. et al. (2008). Restrictive enlargement of the pulmonary annulus at surgical repair of tetralogy of Fallot: 10-year experience with a uniform surgical strategy. Eur J Cardiothorac Surg, Vol.34, (November 2008), pp. 1041-5. 


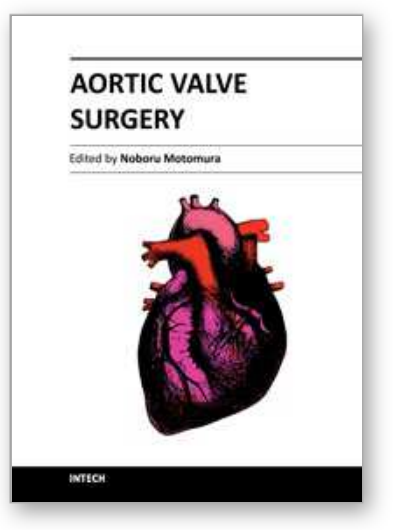

\author{
Aortic Valve Surgery \\ Edited by Prof. Noboru Motomura
}

ISBN 978-953-307-600-3

Hard cover, 246 pages

Publisher InTech

Published online 22, December, 2011

Published in print edition December, 2011

The aortic valve is located at the center of the heart. It is the core of cardiac anatomy and aortic valve surgery has led the field of cardiac surgery. This book describes all aspects of aortic valve surgery and it will help clarify daily questions regarding the clinical practice in aortic valve surgery, as well as induce inspiration and new insights into this field.

\title{
How to reference
}

In order to correctly reference this scholarly work, feel free to copy and paste the following:

Gláucio Furlanetto and Beatriz H. S. Furlanetto (2011). Correction of Transposition of Great Arteries with Ventricular Septal Defect and Left Outflow Tract Obstruction with Double Arterial Translocation with Preservation of the Pulmonary Valve, Aortic Valve Surgery, Prof. Noboru Motomura (Ed.), ISBN: 978-953-307600-3, InTech, Available from: http://www.intechopen.com/books/aortic-valve-surgery/correction-oftransposition-of-great-arteries-with-ventricular-septal-defect-and-left-outflow-tract-

\section{INTECH}

open science | open minds

\section{InTech Europe}

University Campus STeP Ri

Slavka Krautzeka 83/A

51000 Rijeka, Croatia

Phone: +385 (51) 770447

Fax: +385 (51) 686166

www.intechopen.com

\section{InTech China}

Unit 405, Office Block, Hotel Equatorial Shanghai

No.65, Yan An Road (West), Shanghai, 200040, China 中国上海市延安西路65号上海国际贵都大饭店办公楼 405 单元

Phone: +86-21-62489820

Fax: $+86-21-62489821$ 
(C) 2011 The Author(s). Licensee IntechOpen. This is an open access article distributed under the terms of the Creative Commons Attribution 3.0 License, which permits unrestricted use, distribution, and reproduction in any medium, provided the original work is properly cited. 